Var i abl e- gr aspi ng- mode under act uat ed sof t gri pper wi th envi ronment al cont act-based oper at i on

\begin{tabular}{|l|l|}
\hline 著者 & $\begin{array}{l}\text { N shi mur a Toshi hi ro, M zushi ma Kaor i, Suzuki } \\
\text { Yosuke, Tsuj i Tokuo, Wat anabe Tet suyou }\end{array}$ \\
\hline $\begin{array}{l}\text { j our nal or } \\
\text { publ i cat i on t i t l e }\end{array}$ & I EEE Robot i cs and Aut omat i on Let ter s \\
\hline vol une & 2 \\
\hline number & 2 \\
\hline page r ange & 1164 1171 \\
\hline year & $2017-01-31$ \\
\hline URL & ht t p: //hdl . handl e. net /2297/46899 \\
\hline
\end{tabular}




\title{
Variable-grasping-mode underactuated soft gripper with environmental contact-based operation
}

\author{
Toshihiro Nishimura ${ }^{1}$, Student Member, IEEE, Kaori Mizushima ${ }^{1}$, Yosuke Suzuki², \\ Tokuo Tsuji ${ }^{2}$,Member, IEEE, and Tetsuyou Watanabe ${ }^{2}$, Member, IEEE
}

\begin{abstract}
A novel robotic gripper with soft surfaces and underactuated joints was proposed. The soft surface was fabricated from a deformable rubber bag filled with incompressible fluid and a microgripper inside the fluid. A ratchet was installed at the underactuated joint so that the joint's rotation caused by contact with an environment, such as a supporting surface, can be preserved, and the actions of scooping and enveloping an object are realized. With one actuator, the gripper realized three modes i.e., parallel gripper, pinching, and enveloping. The range of graspable objects was wide and included soft, rigid, deformable, fragile, small (boundary length less than 30 $\mathrm{mm}$ ), large (more than $80 \mathrm{~mm}$ long), thin (less than $0.5 \mathrm{~mm}$ ) and heavy (more than $3 \mathrm{~kg}$ ) objects.
\end{abstract}

Index Terms-Grasping, Grippers and Other End-Effectors, Mechanism Design, Soft Material Robotics, Underactuated Robots

\section{INTRODUCTION}

$\mathrm{S}_{\mathrm{f}}^{\mathrm{o}}$ OFTNESS on surfaces or joints of robots is a key feature for realizing versatile and dexterous grasping. Softness can reduce impact at contact, while providing safe interaction with humans and objects and allowing adaptation to object shape. These benefits provide high robustness and the capability to grasp a wide variety of objects to robotic hands. Based on the concept of embedding softness on robotic hands, our group developed a fluid fingertip fabricated from a deformable rubber bag filled with incompressible fluid [1]-[4]. The additional benefits of the fluid fingertip because of its incompressibility are as follows: 1) Contact pressure distribution is uniform. 2) Surface stiffness automatically increases with contact deformation (local passive compliance). The primary drawback of the fluid fingertip is that the weight of graspable objects is low (the maximum graspable weight was less than $0.15 \mathrm{~kg}$ ). Therefore, a microgripper-embedded fluid fingertip was proposed to increase the weight of graspable objects [4]. A parallel gripper was developed to be used with the microgripper-embedded fluid fingertip to ensure the

Manuscript received: September, 10, 2016; Revised December, 5, 2016; Accepted January, 12, 2017.

This paper was recommended for publication by Editor Han Ding upon evaluation of the Associate Editor and Reviewers' comments. This work were partly supported by NEDO Strategic Advancement of Multi-Purpose UltraHuman Robot and Artificial Intelligence Technologies and JSPS KAKENHI Grant Number 16H04298. effectiveness of the fingertip. However, we did not consider real tasks such as picking. As a result, although the gripper succeeded grasping a wide variety of objects, it was not capable of picking up small or thin objects on a table. The next challenge is to develop a robotic hand with microgripperembedded fluid fingertip that can pick up small, thin, fragile, heavy, deformable, soft, rigid, or large objects on a table.

Contact with an environment is part of the basic functionality and working principle of a robotic hand. Here, the environment refers to a supporting surface, other surrounding objects, etc. Intentional contact with the environment rotates underactuated joints and causes the grasping mode to shift, for example, from a parallel gripper mode to an enveloping mode, as illustrated in Fig. 1. This function provides several benefits such as reduction in the number of actuators, convenient fabrication, low cost, and convenient control. Note that another mechanism or function is required to preserve the modified (enveloping) grasping mode even after the robotic gripper leaves the environment.

This paper presents a novel underactuated robotic gripper that considers the abovementioned factors. The main features of this novel gripper are as follows:

- The gripper consists of two fingers, each of which has an underactuated joint and a microgripper-embedded fluid fingertip.

- The range of graspable objects is wide and includes soft, rigid, deformable, fragile, small, large, thin and heavy objects.

- With one actuator, three grasping modes, i.e., parallel gripper, pinching, and enveloping, are realized by utilizing contact with an environment.

- Ratchet mechanisms are installed at underactuated joints to preserve the grasping mode even after the gripper leaves the environment.

${ }^{1}$ T. Nishimura and K. Mizushima are with the Graduated school of Natural science and Technology, Kanazawa University, Kakuma-machi, Kanazawa, 9201192 Japan (e-mail: to.nishimura@stu.kanazawa-u.ac.jp).

${ }^{2}$ Y. Suzuki, T. Tsuji, and T. Watanabe are with the Faculty of Mechanical Engineering, Institute of Science and Engineering, Kanazawa University, Kakuma-machi, Kanazawa, 9201192 Japan (e-mail: te-watanabe@ieee.org).

Digital Object Identifier (DOI): see top of this page. 


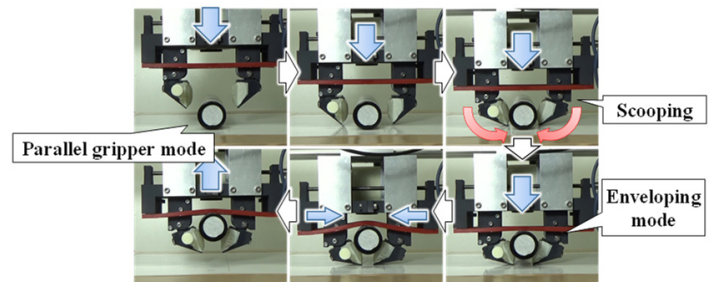

Figure 1. Shift from parallel gripper to enveloping mode by the underactuated soft gripper utilizing contact with a supporting surface. Scooping is included in the procedure.

The following subsection describes the study that produces the abovementioned features. After the gripper design is presented, the details of each grasping mode and control methodologies to realize the three modes are described. Finally, experimental validations are presented.

\section{A. Related works}

Several robotic hands have been developed [5]. Among these, we focus on recently developed robotic hands with softness, which are closely related to this study. Such soft robotic hands can be divided broadly into two types. The first type has softness at joints, e.g., snake-like gripper [6], FRH-4 hand [7], SDM hand [8][9], Pisa-IIT Hand [10][11], SoftHand Pro-D [12], ISR-SoftHand [13], UC-SoftHand [14], Velo Gripper [15], and adaptive prismatic gripper with passive rotational joints [16]. The second type has softness at surfaces, e.g., gel fingertips [17], ISR-SoftHand [13], UC-SoftHand [14], Kanazawa hand [18], deep reef sampling gripper [19], and magnetic fields based gripper [20]. Similar to ISR-SoftHand [13] and UC-SoftHand [14], there are robotic hands that possess both types of softness. Using pneumatic actuators facilitates combination of softness types, for example, in ROBO hand [21][22], Starfish-like hand [23], lightweight underactuated pneumatic fingers [24], soft elastomer quadrupedal robots [25], safe interaction gripper [26], inflatable rubber pockets based gripper [27], puncture resistant soft gripper [28], modular soft robotic grippers [29], and jamming gripper [30][31]. Presence of both types of softness facilitates grasping of several kinds of objects. Here, we compared the two types of softness. The softness at surfaces has superior capability of contact impact reduction and adaptation to object shape, leading to grasping of a wide variety of objects, particularly soft and fragile objects. On the other hand, soft underactuated joints are connected by rigid links and have superior capability of producing large grasping force, leading to grasping of heavy objects. Thus, coexistence of soft and hard material parts can produce a versatile robotic hand that can grasp fragile and heavy objects. The development of the microgripper-embedded fluid fingertips [4] was based on this concept.

Even if a robotic hand does not have sufficient DOFs required for manipulating an object, it can complete the manipulation by making (intentional) contact with the environment and modifying the orientation of the object. Contact with the environment can potentially extend the manipulation capability of robotic hands. Recent studies of motion planning based on this concept are [32]-[35], where motion planning is presented in more detail. Note that motion planning is beyond the scope of this study. A pioneering study of robot hand design that considers contact with the environment is the study of a gripper based on a remote center of compliance [36]. Contact with the environment was effectively utilized to realize grasping at the robotic hands possessing softness [16], [21], [22]. However (based on our knowledge), there is no study in which contact with the environment is intentionally utilized to modify the grasping mode and facilitate the realization of a versatile robotic hand. This study will focus on this challenge.

\section{GRIPPER DESIGN}

\section{A. Design requirements}

The following are the design requirements of the gripper:

1. The gripper must grasp the following objects on a table: (a) soft and hard fragile objects, (b) deformable objects, (c) objects with a boundary length less than 30 $\mathrm{mm},(\mathrm{d})$ objects with a maximum width of $80 \mathrm{~mm}$, (e) objects with a thickness less than $0.5 \mathrm{~mm}$, and (f) objects with a weight more than $3 \mathrm{~kg}$.

2. The gripper must have a single actuator.

3. Three grasping modes, i.e., (a) parallel gripper, (b) pinching, and (c) enveloping, are realized.

Assuming the final application is a shopping register task, we considered a task of picking an item of daily use on a table as the first step. The target items are those that can be found in supermarkets. Their attributes were selected such that they include the items that the previously developed gripper [4] can grasp and those that it cannot. Simplicity is also important for grippers; and we used only one actuator.

As described above, a parallel gripper with microgripperembedded fluid fingertips [4] is a powerful tool for realizing versatile grasping of fragile and heavy objects. We considered utilizing the fingertips in the development of the gripper. The primary issue is that the previous gripper is not capable of handling thin or small objects on a table. To pick up and grasp such objects, the function of scooping is important. Additionally, the enveloping mode is useful for grasping with small forces, as compared to the parallel gripper mode, because in the enveloping mode, the number of contact is larger and the area of contact is wide. The third requirement is based on this analysis.

\section{B. Structure of microgripper-embedded fluid fingertip}

Fig. 2 illustrates a schematic view of the simplified version of the microgripper-embedded fluid fingertips [4]. The fluid fingertip is fabricated from a deformable rubber bag filled with incompressible fluid [1]-[3]. A microgripper is installed inside the fluid. The fingertip can have three "contact modes", as illustrated in Fig. 3. Contact mode 1 is adopted if the object thickness is smaller than the space between the two arms of the microgripper, while contact mode 2 is adopted if the object thickness is larger than the space between the two arms. In both

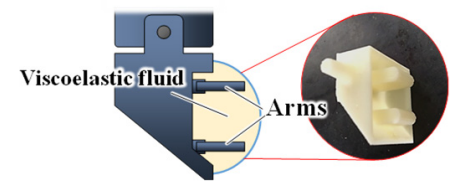

Figure 2. Schematic view of microgripper-embedded fluid fingertip 


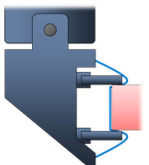

(a) Contact mode 1: The object is pressed into the space between the two arms.

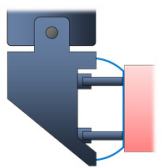

(b) Contact mode 2 The object is held by or is in contact with the two arms.

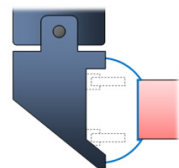

(c) Contact mode 3 : The object is in contact with the fluid part, but not with the two arms.
Figure 3. Three contact modes for the microgripper-embedded fluid fingertip modes, the two arms prevent deformation of the outer rubber and provide large grasping forces. If the weight of object is low, contact with the microgripper is not required. In this case, contact mode 3 is adopted. Contact pressure is uniform and local passive compliance is obtained. Therefore, this mode is appropriate for grasping fragile and complex-shaped objects. Note that the fluid part of the fingertips can reduce impact at contact, absorb strain energy, and adapt to object shape. These benefits are present in contact modes 1 and 2 . In our previous study [4], the two arms were able to rotate and their tips were connected by a flexible and nonexpendable sheet so that an object pinched by the two arms can converge to the center of the sheet and high positioning ability can be obtained. Refer [4] for more details of the structure and functions.

\section{Structure of underactuated soft gripper}

The structure of the underactuated soft gripper is illustrated in Figs. 4 and 5. The distance between the fingers is controlled by a servomotor (Dynamixel XM430-W210) through a screw feed mechanism. A microgripper-embedded fluid fingertip was installed at every fingertip. The fingernail was embedded to realize scooping. Each finger incorporated one underactuated joint so that the parallel gripper and pinching modes are realized. Contact with the environment is utilized to shift the grasping mode. As illustrated in Fig. 4 (a), if the fingertip is pressed against a table or another object, the underactuated joint rotates. To fix the rotation and realize the pinching and enveloping grasps, a ratchet mechanism and a torsion spring were installed at the underactuated joint, as illustrated in Figs. 4 and 5. The fixation of the ratchet is released by fully opening the gripper (Fig. 4 (b)). When the gripper is fully opened, the ratchet pawl makes contact with the releasing pin, and the ratchet is released.

For the enveloping grasp, two separate flexible belts made of silicone were installed as illustrated in Fig. 5. When scooping an object without the belt, there is a possibility of the object moving up and the grasp becoming unstable (as illustrated in the image on the top right). Because of the belts' flexibility, adaptation to object shape was obtained and a stable enveloping grasp was realized. The belts were separated such that a tall object is grasped conveniently in the parallel gripper mode.
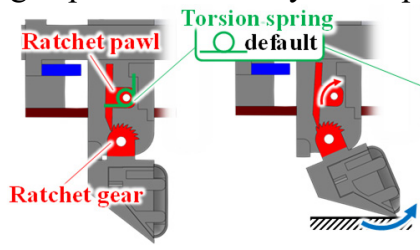
(a) Shift in grasping mode by contact with an (b) Releasing of pawl by
environment such as a supporting surface fully opening the gripper

Figure 4. Schematic view of function of the ratchet installed at an underactuated joint to maintain grasping mode

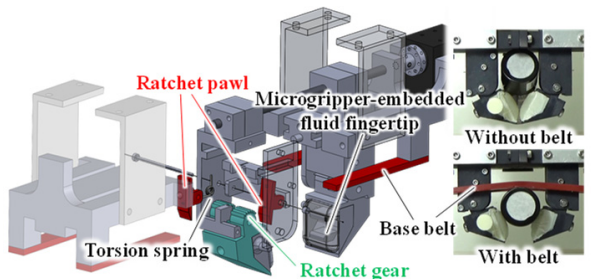

Figure 5. Schematics of the structure of ratchet and flexible base belts

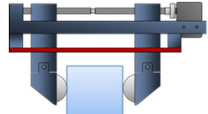

(a) Parallel gripper

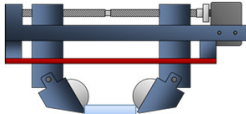

(b) Pinching

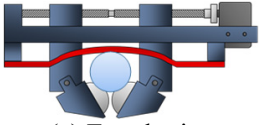

(c) Enveloping
Figure 6. Three variable grasping modes

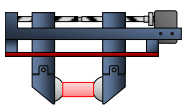

(a) Contact with the microgripperembedded fluid fingertip

Figure 7. Two styles based on the contact area

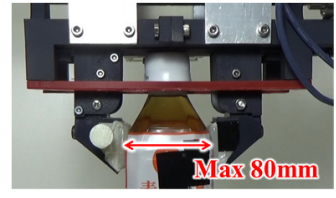

(a-1) Parallel gripper mode; grasp at the fluid fingertip part

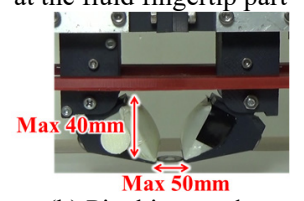

(b) Pinching mode

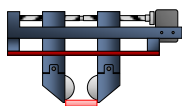

(b) Contact with the tip

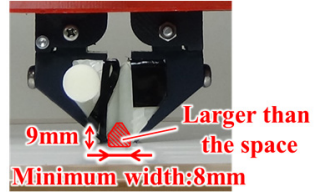

(a-2) Parallel gripper mode; grasp at the tip part

(c) Enveloping mode

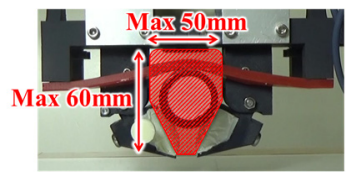

Figure 8. Allowable size of objects in each grasping mode

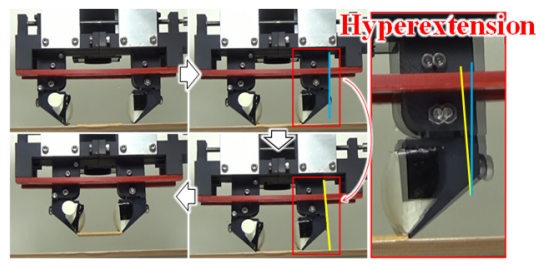

Figure 9. Procedure for grasping a thin and rigid object utilizing hyperextending motion with parallel gripper mode

\section{GRASPING MODE}

Using the design described in the previous section, the three grasping modes illustrated in Fig. 6 are realized by utilizing contact with the environment. Note that these are the primary modes; however, a combination of two modes or a partially realized mode (for example, only one finger is rotated) can be also realized. The three grasping modes are described below.

\section{A. Parallel gripper mode; Fig. 6 (a)}

There are two styles of contact (Fig. 7) depending on the contact area. If the object is sufficiently tall for it to make contact with the fluid fingertip area, it is grasped at the fluid fingertip area. The features of this style are as follows:

- The functions of the fluid fingertip (described in section II B) are fully utilized and a wide variety of objects are grasped. 
- The maximum width of graspable objects is $80 \mathrm{~mm}$ (Fig. 8 (a-1)).

Otherwise, the object is grasped at the tips. The features of this style are as follows:

- Small and thin objects, whose size is larger than the space shown in Fig. 8 (a-2), are pinched.

- Because of passive compliance of the finger, the fingertip is hyperextended (Fig. 9) and the object is pinched.

\section{B. Pinching mode; Fig 6 (b)}

The features of this mode are as follows:

- Scooping is realized and small or thin objects on a table are picked up and grasped.

- Soft and fragile objects are grasped by utilizing the microgripper-embedded fluid fingertip part.

- The ratchet has backlash; therefore, a control methodology considering the backlash is required.

- The size of graspable objects is shown in Fig. 8 (b).

\section{Enveloping mode; Fig 6 (c)}

This mode is adopted when attempting to pick up an object in the pinching mode and the object makes contact with the base belt (Fig. 5). The features of this mode are as follows:

- Scooping is realized.

- Geometrical constraints are applied through multiple and widely spread contact points, and soft and fragile objects are grasped with small contact forces.

- Gripper size limits the size of graspable objects (Fig. 8 (c)).

\section{ProcedURES FOR GRASPING}

In this section, we present the procedure for grasping objects by employing the gripper developed in this study, following the grasping modes illustrated in Fig. 6. When grasping an object in the parallel gripper mode and the style illustrated in Fig. 7 (a), the gripper is controlled such that the object can be grasped at the microgripper-embedded fluid fingertip parts. When the style illustrated in Fig. 7 (b) is required, contact with a table is utilized (Fig. 9). First, the fingertips make contact with the table; then, the gripper is closed. If the object makes contact with the fingernail, the fingertip is hyperextended. For picking up the object in this state, it is grasped at the area between the nail and the fluid fingertip. It is necessary to note that it is difficult to grasp objects at the exact nail tip because of marginal friction.

If the pinching or enveloping grasping mode is required, an appropriate procedure has to be followed. First, the fingertips make contact with the supporting surface and rotate their joints. Then, the gripper is closed, and it scoops a target object. Finally, the object is picked up. If the object makes contact with the base belt illustrated in Fig. 5, the enveloping mode is adopted. When rotating the fingertips, the rotated joint angle is fixed by the ratchet illustrated in Figs. 4 and 5. However, a recess in the gear causes backlash in the ratchet, and the gripper is opened by the same amount as that of the backlash during the picking. Therefore, we regulate the closing distance of the gripper when

picking up an object. The basic strategy is to maintain the distance between the nails of the fingertips constant when picking up the object.

We consider the state in which the fingertip initially makes contact with the supporting surface to be the reference state, and set the coordinate frame as illustrated in Fig. 10 (a). The nomenclature is presented in Fig. 10.

Considering manufacturing and fabrication errors, the amount of backlash was experimentally evaluated. The gripper was pressed against the table by controlling the pressing distance, $z[\mathrm{~mm}]$. There are three teeth on the ratchet and three lock-switching angles. We examined $z[\mathrm{~mm}]$ three times for every angle, where the locking switches. Table I presents the results. Because of manufacturing and fabrication errors, the values of $z[\mathrm{~mm}]$ for lock switching for the right and left fingertips were different. Table II shows the range of $z[\mathrm{~mm}]$ where the gear-lock states of both fingertips are identical. The range was derived by assuming that gear lock occurs within $\bar{z}_{n} \pm \sigma_{n}$ (Table I).

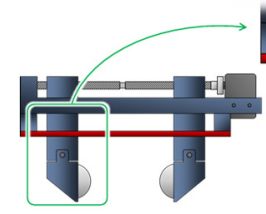

(a) Reference state where the fingertip initially makes contact with the supporting surface

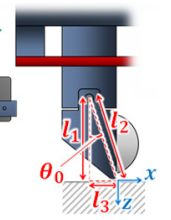

igure 10. Nom

Nomenclature for analysis of backlash at the ratchet

TABLE I. EXPERIMENTAL RESULTS OF PRESSING DISTANCE $Z$ [MM] WHERE LOCKING OF THE RATCHET GEAR SWITCHED.

\begin{tabular}{|c|c|c|c|c|c|c|}
\hline \multirow{2}{*}{ Gear lock } & \multicolumn{3}{|c|}{ Right fingertip } & \multicolumn{3}{|c|}{ Left fingertip } \\
\cline { 2 - 7 } & 1st & 2nd & 3rd & 1st & 2nd & 3rd \\
\hline \hline Mean $\left(\bar{z}_{n}[\mathrm{~mm}]\right)$ & 6.43 & 10.3 & 15.2 & 5.93 & 10.9 & 14.9 \\
\hline $\begin{array}{c}\text { Standard } \\
\text { deviation }\left(\sigma_{n}\right. \\
[\mathrm{mm}])\end{array}$ & 0.18 & 0.32 & 0.05 & 0.21 & 0.21 & 0.33 \\
\hline
\end{tabular}

TABLE II. RANGE OF PRESSING DISTANCE $Z$ [MM] WHERE THE GEARLOCK STATES OF BOTH FINGERTIPS ARE IDENTICAL.

\begin{tabular}{|c|c|}
\hline Gear-lock state & $\begin{array}{c}\text { Range of } z[\mathrm{~mm}] \text { where the gear-lock states } \\
\text { of both fingertips are identical }\end{array}$ \\
\hline \hline 1st gear lock & $6.61-9.98$ \\
\hline 2nd gear lock & $11.1-14.6$ \\
\hline 3rd gear lock & $15.3-$ \\
\hline
\end{tabular}

TABLE III. PRESSING DISTANCE $Z$ [MM] IN THE CASE WHERE ROTATION DUE TO BACKLASH OF THE RATCHET STOPS WHEN MOVING UP THE GRIPPER.

\begin{tabular}{|c|c|c|}
\hline $\begin{array}{c}\text { Initial state of the } \\
\text { ratchet before the } \\
\text { gripper moves } \\
\text { upward }\end{array}$ & Mean z $[\mathrm{mm}]$ & $\begin{array}{c}\text { Standard deviation } \\
{[\mathrm{mm}]}\end{array}$ \\
\hline \hline 1st gear lock & 4.46 & 0.12 \\
\hline 2nd gear lock & 7.87 & 0.16 \\
\hline 3rd gear lock & 11.9 & 0.32 \\
\hline
\end{tabular}

Because of backlash of the ratchet, the joint angle returns when the gripper leaves the supporting surface, and the gripper opens. There is a possibility of the object falling down because of the opening motion. We investigated the return angle of the ratchet. Initially, we set $z[\mathrm{~mm}]$ such that it is within any of the ranges listed in Table II (both gear-lock states were identical). 
Then, we moved the gripper upward and measured $z$ [mm] when the return rotation of the joint angle stopped. Table III presents the result.

By controlling the speeds of lifting $\dot{z}$ and closing $\dot{x}$, we resolved the issue of opening of the gripper because of backlash of the ratchet when lifting the gripper (in the pinching or enveloping mode). Consider the case in which the gripper moves downward by $z$, as illustrated in Fig. 10 (b). We obtain

$$
l_{1}^{2}+l_{3}^{2}=\left(l_{1}-z\right)^{2}+\left(x+l_{3}\right)^{2}
$$

By differentiating (1) with respect to time, we obtain

$$
\dot{z}=\dot{x} \tan \theta
$$

where

$$
\tan \theta=\frac{\sqrt{l_{2}^{2}-\left(l_{1}-z\right)^{2}}}{l_{1}-z}
$$

(2) indicates the process by which the fingertips open because of lifting of the gripper; it is the relationship between the speeds of opening of the fingertips $(-\dot{x})$ and lifting of the gripper $(\dot{z})$. Let $v_{\text {grip }}$ be the speed of closing the gripper. Then, by controlling $\dot{z}$ such that $\dot{z}=\dot{x} \tan \theta=v_{\text {grip }} \tan \theta(\dot{x}=$ $\left.v_{\text {grip }}\right)$, the distance between the tips is maintained constant during lifting of the gripper, irrespective of joint rotation due to backlash of the ratchet. Note that $\tan \theta$ is a function of $z$. Moreover, in the developed gripper, $l_{1}=44.2 \mathrm{~mm}, l_{2}=46.5$ $\mathrm{mm}, l_{3}=14.3 \mathrm{~mm}$, and $\theta_{0}=17.9^{\circ}$.

Fig. 11 illustrates a summary of the control schema for grasping an object with the pinching or enveloping mode. First, the lowering distance determined by object size determines the number of gear-lock, and $z[\mathrm{~mm}]$ is controlled such that it is within any of the ranges listed in Table II (both gear-lock states are identical).

Next, we select the value of $z[\mathrm{~mm}]$ from Table III according to the gear-lock states, and set it to be $z_{c r}[\mathrm{~mm}]$, which is the distance above which it is necessary to control the closing distance of the gripper. Then, the gripper moves upward with speed $\dot{z}=v_{\text {grip }} \tan \theta$ while being closed at speed $v_{\text {grip }}$, until $z$ [mm] becomes equal to $z_{c r}[\mathrm{~mm}]$. When $z$ [mm] becomes equal to $z_{c r}[\mathrm{~mm}]$, we stop closing the gripper and move it upward to the desired state. Here, we set $v_{\text {grip }}=1.2 \mathrm{~mm} / \mathrm{s}$. Fig. 12 provides an overview of the actual application of this procedure. The blue line implies that the closing distance was maintained constant.

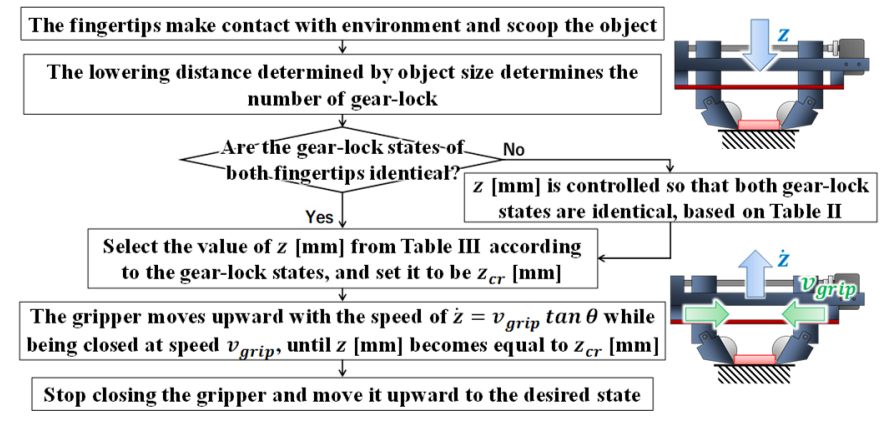

Figure 11. Control schema for grasping an object with pinching or enveloping mode

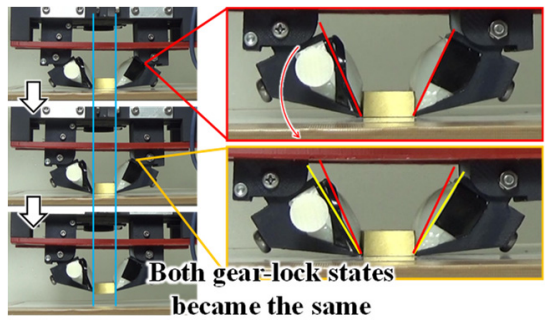

Figure 12. Example of application of the control schema illustrated in Fig. 11 (grasping an object with pinching mode)

\section{EXPERIMENTAL VALIDATION}

To evaluate the capability of the developed robotic gripper, several experimental tests were conducted.

\section{A. Picking up and grasping an object on a table}

We conducted tests of grasping a wide variety of objects on a table, and evaluated the efficacy of each grasping mode. The closing distance, the grasping mode, and the lowering distance were predefined manually while the control schema shown in Fig. 11 was adapted in an automatic manner. When grasping fragile objects such as tofu, the closing distance was manually determined based on the methods presented in [1]-[3]. The objects were randomly placed on the table manually, such that the center of the objects was around the depicted circle with 5 $\mathrm{mm}$ radius. Then, the object position had cumulative deviation resulting from manual placement and the radius of the circle. Fig. 13 illustrates the representative examples of the grasping test. Table IV shows all results and success rates (see also attachment video for the full results).

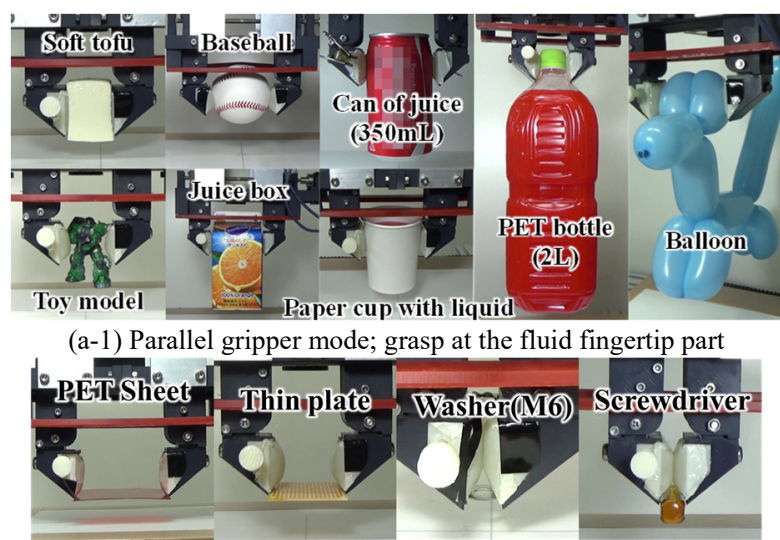

(a-2) Parallel gripper mode; grasp at the tip part

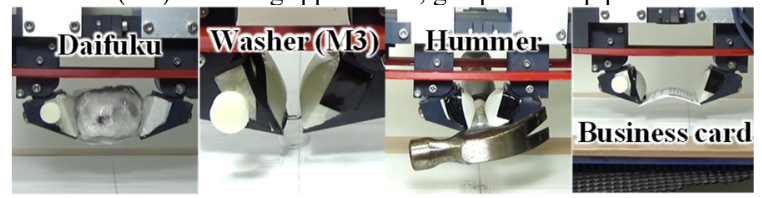

(b) Pinching mode

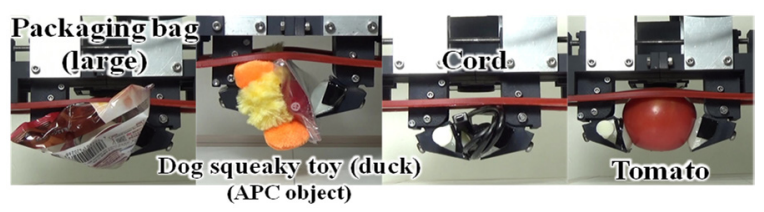

(c) Enveloping mode

Figure 13. Representative results of grasping test 
TABLE IV. GRASPING TEST RESULTS WITH SUCCESS RATE (RED VALUES CORRESPOND TO THE WIDTH OF OBJECT IN THE OPENING DIRECTION OF GRIPPER

\begin{tabular}{|c|c|c|c|c|c|c|c|}
\hline $\begin{array}{l}\text { Success } \\
\text { rate }\end{array}$ & \multicolumn{3}{|c|}{ Parallel gripper mode } & \multicolumn{2}{|c|}{ Pinching mode } & \multicolumn{2}{|c|}{ Enveloping mode } \\
\hline \multirow{13}{*}{$3 / 3$} & $\begin{array}{c}\text { Pencil cup } \\
\text { (Thickness:4) }\end{array}$ & $\begin{array}{c}\text { Dove bar }{ }^{1} \\
(94 \times 65 \times 35)\end{array}$ & $\begin{array}{l}\text { Outlet plug covers }^{1} \\
(\mathbf{6 8} \times 187 \times 30)\end{array}$ & $\begin{array}{c}\text { Dice }^{2} \\
(\mathbf{1 6} \times 16 \times 16) \\
\end{array}$ & $\begin{array}{l}\text { Bolt (M3) } \\
(\varnothing \mathbf{5 . 5} \times 13)\end{array}$ & $\begin{array}{c}\text { Tomato } \\
(\varnothing 70)\end{array}$ & $\begin{array}{c}\text { Tennis ball }^{2} \\
(\varnothing 64.7)\end{array}$ \\
\hline & $\begin{array}{c}\text { Board eraser } \\
(\mathbf{5 5} \times 132 \times 35)\end{array}$ & $\begin{array}{c}\text { Thin plate } \\
(72 \times \mathbf{4 7} \times 1.6)\end{array}$ & $\begin{array}{c}\text { Cups }^{1} \\
(\varnothing 67 \times 158)\end{array}$ & $\begin{array}{c}\text { Soft tofu } \\
(26 \times 26 \times 30)\end{array}$ & $\begin{array}{c}\text { Washer (M3) } \\
(\emptyset 6 \times 0.5)\end{array}$ & $\begin{array}{l}\text { Glass bottle } \\
(\emptyset \mathbf{5 1} \times 135)\end{array}$ & $\begin{array}{c}\text { Dog squeaky toy }(\text { Duck })^{1} \\
(\mathbf{5 0} \times 97)\end{array}$ \\
\hline & $\begin{array}{l}\text { Golf ball }^{2} \\
(\varnothing \mathbf{4 2 . 7})\end{array}$ & $\begin{array}{c}\text { Soft tofu } \\
(26 \times 26 \times 30)\end{array}$ & $\begin{array}{c}\text { Baseball }^{2} \\
(\varnothing \mathbf{8 0})\end{array}$ & $\begin{array}{c}\text { Battery } \\
(\varnothing \mathbf{1 4 . 5} \times 50.5) \\
\end{array}$ & $\begin{array}{c}\text { PET sheet } \\
(100 \times \mathbf{7 0} \times 1) \\
\end{array}$ & $\begin{array}{c}\text { Bath toy }^{1} \\
(\mathbf{9 0} \times 133 \times 71) \\
\end{array}$ & $\begin{array}{c}\text { Dog squeaky toy }(\text { Frog })^{1} \\
(\mathbf{3 0} \times 116)\end{array}$ \\
\hline & $\begin{array}{c}\text { Tennis ball }^{1} \\
(\varnothing \mathbf{6 3} \times 108 \times 190) \\
\end{array}$ & $\begin{array}{c}\text { Spark plug }{ }^{1} \\
(96 \times \mathbf{2 5} \times 22) \\
\end{array}$ & $\begin{array}{c}\text { Spam can }{ }^{2} \\
(\mathbf{5 0} \times 97 \times 82)\end{array}$ & $\begin{array}{l}\text { Daifuku } \\
(\varnothing \mathbf{5 0})\end{array}$ & $\begin{array}{c}\text { Toy model } \\
(60 \times \mathbf{4 0} \times 30)\end{array}$ & $\begin{array}{c}\text { Banana } \\
(\varnothing \mathbf{3 5} \times 150)\end{array}$ & $\begin{array}{l}\text { Ball dog toys }^{1} \\
(67 \times 123)\end{array}$ \\
\hline & $\begin{array}{l}\text { Tomato can } \\
(\varnothing 66 \times 101) \\
\end{array}$ & $\begin{array}{c}\text { Screwdriver } \\
(\mathbf{1 3} \times 13 \times 130)\end{array}$ & $\begin{array}{l}\text { PET bottle (2L) } \\
\text { (cap: } \varnothing \mathbf{3 5})\end{array}$ & \begin{tabular}{|c|} 
Business card \\
$(91 \times \mathbf{5 5} \times 0.2)$ \\
\end{tabular} & $\begin{array}{l}\text { Washer (M6) } \\
(\varnothing \mathbf{1 1 . 5} \times 1.6) \\
\end{array}$ & $\begin{array}{c}\text { Onion } \\
(70 \times \varnothing 55)\end{array}$ & $\begin{array}{c}\text { Plastic strawberry }{ }^{2} \\
(\mathbf{4 3 . 8} \times 55)\end{array}$ \\
\hline & $\begin{array}{l}\text { Washer(M6) } \\
(\varnothing \mathbf{1 1 . 5} \times 1.6) \\
\end{array}$ & $\begin{array}{c}\text { Battery } \\
(\varnothing \mathbf{1 4 . 5} \times 50.5)\end{array}$ & $\begin{array}{c}\text { Balloon } \\
(250 \times \mathbf{6 0} \times 150)\end{array}$ & $\begin{array}{c}\text { Plier } \\
(29 \times 135 \times 15) \\
\end{array}$ & $\begin{array}{l}\text { Hummer } \\
(\varnothing \mathbf{1 4} \times 250)\end{array}$ & $\begin{array}{c}\text { Rope }^{2} \\
(8 \times 2000)\end{array}$ & $\begin{array}{l}\text { Packaging bag (large }) \\
(\mathbf{7 0} \times 200 \times 40)\end{array}$ \\
\hline & $\begin{array}{c}\text { Screwdriver set }{ }^{1} \\
(77 \times 137)\end{array}$ & $\begin{array}{l}\text { Racquetball }^{2} \\
(\varnothing 55.3)\end{array}$ & $\begin{array}{l}\text { Tablet pill box } \\
(47 \times 78 \times 7)\end{array}$ & \begin{tabular}{|c|} 
Screwdriver \\
$(\mathbf{1 3} \times 13 \times 130)$ \\
\end{tabular} & $\begin{array}{c}\text { Glue }^{1} \\
(29 \times \mathbf{6 3} \times 146) \\
\end{array}$ & $\begin{array}{c}\text { Cord } \\
(8 \times 1000) \\
\end{array}$ & $\begin{array}{l}\text { Packaging bag (small) } \\
(\mathbf{5 0} \times 150 \times 30)\end{array}$ \\
\hline & $\begin{array}{c}\text { Pen } \\
(\varnothing \mathbf{1 2} \times 139)\end{array}$ & $\begin{array}{c}\text { Paper cup with } \\
\text { liquid } \\
(\mathbf{6 8} \times 187 \times 30) \\
\end{array}$ & $\begin{array}{l}\text { Plastic strawberry } \\
\quad(\mathbf{4 3 . 8} \times 55)\end{array}$ & $\begin{array}{l}\text { Binder clip } \\
(\mathbf{2 0} \times 37 \times 9)\end{array}$ & $\begin{array}{l}\text { Ball point pen } \\
\text { refill } \\
(\mathbf{2 5} \times 134) \\
\end{array}$ & $\begin{array}{c}\text { Carrot } \\
(\varnothing \mathbf{4 5} \times 180)\end{array}$ & $\begin{array}{l}\text { Packaged bread } \\
(\mathbf{6 5} \times 180 \times 30)\end{array}$ \\
\hline & $\begin{array}{l}\text { Small spray } \\
(\varnothing \mathbf{4 0} \times 150)\end{array}$ & $\begin{array}{c}\text { Toy model } \\
(60 \times \mathbf{4 0} \times 30)\end{array}$ & $\begin{array}{c}\text { Dental treats }{ }^{1} \\
(210 \times \mathbf{5 4} \times 160) \\
\end{array}$ & $\begin{array}{c}\text { Japanese coin } \\
(\varnothing \mathbf{2 2} \times 1)\end{array}$ & $\begin{array}{c}\text { Chocolate } \\
(\varnothing \mathbf{2 7})\end{array}$ & $\begin{array}{l}\text { Small spray } \\
(\varnothing \mathbf{4 0} \times 150)\end{array}$ & $\begin{array}{c}\text { Electric bulb } \\
\text { (base: } \varnothing \mathbf{3 5} \times 40 \text { ) }\end{array}$ \\
\hline & $\begin{array}{c}\text { Bottle brush }^{1} \\
(115 \times \mathbf{5 5} \times 290) \\
\end{array}$ & $\begin{array}{c}\text { Large book }^{1} \\
(125 \times 198 \times \mathbf{1 2})\end{array}$ & $\begin{array}{c}\text { Small book }{ }^{1} \\
(113 \times 170 \times \mathbf{8})\end{array}$ & & & $\begin{array}{l}\text { Large Spray } \\
(\varnothing \mathbf{6 6} \times 150) \\
\end{array}$ & $\begin{array}{l}\text { Safety glasses }{ }^{1} \\
(\mathbf{6 5} \times 50 \times 190)\end{array}$ \\
\hline & $\begin{array}{c}\text { Color pen set }^{1} \\
(118 \times 120 \times \mathbf{2 0}) \\
\end{array}$ & $\begin{array}{c}\text { Juice box } \\
(\mathbf{7 0} \times 140) \\
\end{array}$ & $\begin{array}{c}\text { PET bottle }(500 \mathrm{~mL}) \\
(\mathbf{7 0} \times 70 \times 190)\end{array}$ & & & $\begin{array}{c}\text { Paprika } \\
(\varnothing \mathbf{5 5} \times 85) \\
\end{array}$ & \\
\hline & $\begin{array}{c}\text { PET sheet } \\
(100 \times \mathbf{7 0} \times 0.45)\end{array}$ & $\begin{array}{l}\text { Can of juice } \\
(\emptyset 66 \times 122)\end{array}$ & $\begin{array}{l}\text { Self-stick notes }^{1} \\
(\mathbf{5 1} \times 116 \times 39) \\
\end{array}$ & & & & \\
\hline & $\begin{array}{c}\text { Crayon }^{1} \\
(145 \times \mathbf{3 5} \times 126) \\
\end{array}$ & $\begin{array}{l}\text { Large Spray } \\
(\varnothing 6 \mathbf{6 6} \times 150)\end{array}$ & $\begin{array}{c}\text { Tomato } \\
(\varnothing 70)\end{array}$ & & & & \\
\hline $1 / 3$ & \multicolumn{2}{|c|}{$\begin{array}{c}\text { Index cards }^{1} \\
(76 \times 128 \times 20)\end{array}$} & $\begin{array}{l}\text { Pringles can } \\
(\varnothing 75 \times 250) \\
(\varnothing 5\end{array}$ & & \multicolumn{2}{|r|}{ - } \\
\hline $0 / 3^{3}$ & \multicolumn{2}{|c|}{$\begin{array}{c}\text { Bottle brush }{ }^{1} \\
(115 \times 55 \times 290)\end{array}$} & $\begin{array}{c}\text { Large book }{ }^{1} \\
(\mathbf{1 2 5} \times 198 \times 12)\end{array}$ & \multicolumn{2}{|r|}{$\begin{array}{c}\text { Small book }^{1} \\
(\mathbf{1 1 3} \times 170 \times 8)\end{array}$} & \multicolumn{2}{|r|}{$\begin{array}{c}\text { Color pens }{ }^{1} \\
(118 \times 120 \times 20) \\
\end{array}$} \\
\hline
\end{tabular}

1: Amazon picking challenge objects, 2: YCB objects, $\quad 3$ : By changing the pose, these objects were grasped with a success rate of $3 / 3$

\section{B. Feasibility analysis and test}

Here, the maximum weight of graspable objects was investigated. Fig. 14 illustrates the model for the analysis. When adopting the parallel gripper mode, the stopper illustrated in Fig. 14 (a) is utilized to generate large grasping forces. The tip area is meant for grasping thin and small objects. Therefore, the case in which the microgripper-embedded fluid fingertip part is utilized is the problem. When grasping heavy objects with the fluid fingertip part, the microgipper is utilized. Then, we analyzed the most critical case illustrated in Fig. 14 (a), where an external force is exerted at the tip of the lower arm in the vertical direction. The lower arm is modeled by a cantilever beam. Let $f_{a}$ be the allowable load exerted at the tip of the lower arm. Then, $f_{a}$ is expressed by

$$
f_{a}=\frac{\sigma_{A B S}}{h_{a}} Z_{a}=\frac{\sigma_{A B S}}{h_{a}} \frac{b_{a} e_{a}^{2}}{6}
$$

where $\sigma_{A B S}$ is the flexural strength of the material (ABS plus plastic (Stratasys) was utilized; $\sigma_{A B S}=35 \mathrm{MPa}$ ), $b_{a}=13 \mathrm{~mm}$, $e_{a}=5 \mathrm{~mm}, h_{a}=13 \mathrm{~mm}$ are the dimensions of the arm, and $Z_{a}$ is the section modulus. Then, $f_{a}=150 \mathrm{~N}$ (15 kgf). Moreover, it can be observed that the gripper can grasp an object with a weight of more than $3 \mathrm{~kg}$ (design requirement 1 (f)) if the motor has the required power capacity and there is adequate amount of friction.

Next, we consider the allowable load in the pinching and enveloping modes. In these cases, the amount of load that the ratchet can withstand is the most important factor. Therefore, we consider the model illustrated in Fig. 14 (b), assuming that the maximum stress occurs at the gear tooth. In this case, the allowable torque at the joint is expressed by

$$
\tau_{g}=r_{\text {gear }} \frac{\sigma_{A B S}}{h_{g}} Z_{g}=r_{\text {gear }} \frac{\sigma_{A B S}}{h_{g}} \frac{b_{g} e_{g}^{2}}{6}
$$

where $b_{g}=12 \mathrm{~mm}, e_{g}=1.57 \mathrm{~mm}, h_{g}=2 \mathrm{~mm}$ are the dimensions of the tooth and $r_{\text {gear }}=8 \mathrm{~mm}$. Therefore, $\tau_{g}=$ $0.69 \mathrm{Nm}$. If the operating point of the load is the tip of the fingertip illustrated in Fig. 14 (b), then the allowable load is 22 $\mathrm{N}(2.2 \mathrm{kgf})$. If both fingertips are utilized, then the total allowable load (weight) is $44 \mathrm{~N}$ (4.4 kgf). Moreover, it can be observed that the gripper can grasp an object with a weight of more than $3 \mathrm{~kg}$ in these modes. To confirm the validity of this analysis, we conducted FEM analysis, where the derived allowable torque $\left(\tau_{g}=0.69 \mathrm{Nm}\right)$ was applied. Fig. 15 presents the results for the setting in which the model type was an isotropic elastic medium, Young's modulus was $2.4 \mathrm{GPa}$, and Poisson's ratio was 0.39 . The calculated stress was the highest at the tooth, and its magnitude was $34 \mathrm{MPa}$, which is approximately equal to the flexural strength, $\sigma_{A B S}$. The result validated the above analysis.

Additionally, a feasibility test was conducted to determine whether the maximum weight of graspable objects is more than 
$3 \mathrm{~kg}$ in every mode. We investigated the parallel gripper mode and the case in which the ratchet gear at the joint was locked once. The gripper grasped a thin plate (universal substrate), and the plate was pulled by a force gauge (IMADA DS2-500N) until the magnitude of the load measured by the force gauge exceeded $30 \mathrm{~N}$ ( $3 \mathrm{kgf})$. Fig. 16 presents the results where the gripper restrained the applied load in both cases. Moreover, we can conclude that the gripper is capable of grasping an object whose weight is equal to or less than $3 \mathrm{~kg}$ with any mode illustrated in Fig. 6.

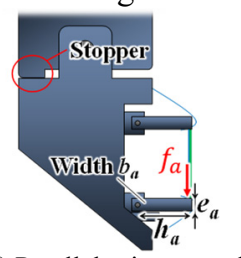

(a) Parallel gripper mode

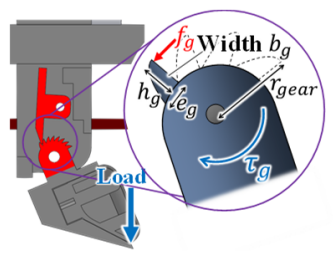

(b) Pinching or enveloping mode
Figure 14. Models for feasibility analysis

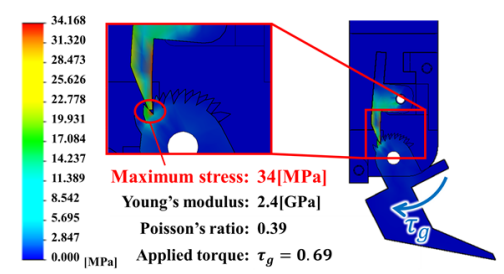

Figure 15. FEM analysis to determine the point where maximum stress occurs at the ratchet and the value of the maximum stress

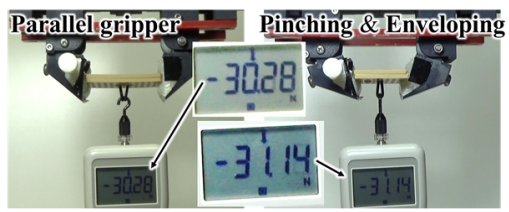

Figure 16. Results for feasibility test to evaluate whether the maximum weight of graspable objects is more than $3 \mathrm{~kg}$

\section{Discussion}

As observed from Figs. 1, 9, 12, and 13, the developed gripper is capable of grasping a wide variety of objects. First, we determine whether the design requirements are satisfied. We observe that the design requirements 1(f), 2, and 3 (section II.A) were satisfied. Then, the design requirements 1 (a)-(e) are evaluated. Examples of soft and fragile objects that were grasped are a paper cup with liquid, soft tofu, and daifuku (Japanese sweet). The fluid fingertips were effective for grasping these objects. Examples of hard and fragile objects that were grasped are a toy model, a glass bottle, an electric bulb, and a thin plate. If the object has complex shape, the fluid fingertip adapts to the shape. If the object is thin, the functions of scooping and hyperextending are carried out effectively. Next, deformable objects, such as packaging bags and cords, were grasped by adopting the enveloping mode. This mode can reduce the required force for grasping. Therefore, the mode functioned effectively while grasping deformable objects. Thin (thickness less than $0.5 \mathrm{~mm}$ ) and deformable objects, such as a business card made of paper and a PET sheet, were grasped at the tips. Either the scooping or the hyperextending function was utilized. Long objects of a length more than $80 \mathrm{~mm}$, such as a balloon, a PET bottle, and a juice box, were grasped by adopting the parallel gripper mode. A representative example of small objects with a boundary length of less than $30 \mathrm{~mm}$ is a M3 washer (width $7 \mathrm{~mm}$ ). The parallel gripper mode could not be adopted because of the space under the fluid fingertips, as illustrated in Fig. 8 (a-2). A width of $8 \mathrm{~mm}$ is required to grasp an object with the parallel gripper mode. For such an object, the scooping function is effective, and the pinching mode was selected. Fig. 8 illustrates the allowable size of objects for every grasping mode, namely, the graspable size of the developed gripper. This is the primary limitation, and the grasping failed frequently when the object size was close to the size limitation (see Table IV). The other limitations are that the grasping mode cannot change on a supporting surface where the fingernails cannot slide (another method should be adapted), or we cannot obtain a sufficiently large reaction force $(2.5 \mathrm{~N})$ to rotate the passive joints. Fig. 17 presents the recommended procedure for selecting the grasping mode. This is a recommendation because, for example, it is possible that an object (such as the tomato illustrated in Fig. 13 (c)) that can be grasped with the enveloping mode, can be also grasped with the parallel gripper mode. The enveloping mode can reduce grasping force, and we recommend this mode.

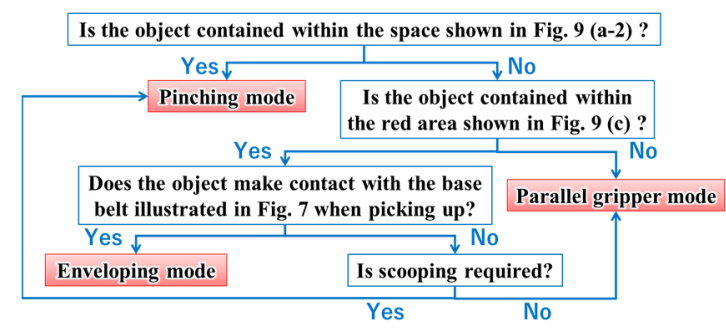

Figure 17. Recommended procedure for selecting grasping mode

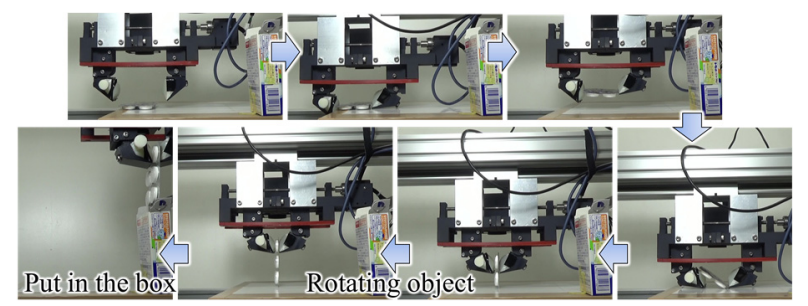

Figure 18. Examples of manipulation by the developed gripper, based on manual motion planning (Inserting a thin and fragile object into a paper box, utilizing desk edge)

It is necessary to note that a combination of the grasping modes can be adopted. For example, a juice box (the second column of the second row of Fig. 13 (a-1)) was grasped with a combination of the parallel gripper and enveloping modes. If motion planning and environments other than a flat area of a table are utilized, the range of the grasping modes can be extended. Fig. 18 illustrates an example of such combinations; however, manual planning was adopted. The examples illustrate the possibility of application of the developed gripper in dexterous manipulation.

\section{CONCLUSION}

This paper presented a novel robotic gripper that can change grasping modes by utilizing contact with an environment. The 
changeable grasping modes are the parallel gripper, pinching, and enveloping modes. Microgripper-embedded fluid fingertips [4] were used to realize versatile grasping. The fingertip has a passive joint with a ratchet and a torsion spring. The ratchet has the function of fixing the joint angle, which is passively rotated through contact with the environment. The fixation of the ratchet is released by fully opening the gripper. By incorporating these mechanisms, the developed gripper functions with one actuator and has passive compliance at the surface and the joint. Utilizing the three grasping modes, the gripper realized the action of picking up a wide variety of objects on a table. The range of graspable objects is wide and includes soft, rigid, deformable, fragile, small (boundary length less than $30 \mathrm{~mm}$ ), large (more than $80 \mathrm{~mm}$ long), thin (less than $0.5 \mathrm{~mm}$ ), and heavy (more than $3 \mathrm{~kg}$ ) objects. A few of our future works focus on grasping and motion planning based on sensor data or optimal design of size and material based on actual tasks. Identifying and automizing manually determined factors are the keys for automatic grasping and manipulation.

\section{REFERENCES}

[1] R. Maruyama, T. Watanabe, and M. Uchida, "Delicate grasping by robotic gripper with incompressible fluid-based deformable fingertips," IEEE Int. Conf. Intell. Robot. Syst., pp. 5469-5474, 2013.

[2] R. Adachi, Y. Fujihira, and T. Watanabe, "Identification of danger state for grasping delicate tofu with fingertips containing viscoelastic fluid," IEEE/RSJ Int. Conf. Intell. Robot. Syst., no. Sept 28-Oct 2, pp. 497-503, 2015.

[3] T. Nishimura, Y. Fujihira, R. Adachi, and T. Watanabe, "New Condition for Tofu Stable Grasping with Fluid Fingertips," Proc. IEEE Int. Conf. Autom. Sci. Eng., 2016.

[4] T. Nishimura, Y. Fujihira, and T. Watanabe, "Microgripper-embedded fluid fingertipenhancing positioning and holding abilities for universal grasping," J. Mech. Robot, submitted for publication.

[5] M. Controzzi, C. Cipriani, and M. C. Carrozza, "Design of Artificial Hands: A Review," 2014, pp. 219-246.

[6] S. Hirose and Y. Umetani, "The development of soft gripper for the versatile robot hand," Mech. Mach. Theory, vol. 13, no. 3, pp. 351-359, 1978.

[7] I. Gaiser, S. Schulz, A. Kargov, H. Klosek, A. Bierbaum, C. Pylatiuk, R. Oberle, T. Werner, T. Asfour, G. Bretthauer, and R. Dillmann, "A new anthropomorphic robotic hand," in Humanoids 2008 - 8th IEEE-RAS International Conference on Humanoid Robots, 2008, no. August 2016, pp. $418-422$.

[8] A. M. Dollar and R. D. Howe, "The Highly Adaptive SDM Hand: Design and Performance Evaluation," Int. J. Rob. Res., vol. 29, no. 5, pp. 585-597, Apr. 2010.

[9] L. U. Odhner, L. P. Jentoft, M. R. Claffee, N. Corson, Y. Tenzer, R. R. Ma, M. Buehler, R. Kohout, R. D. Howe, and A. M. Dollar, "A compliant, underactuated hand for robust manipulation," Int. J. Rob. Res., vol. 33, no. 5, pp. 736-752, Apr. 2014.

[10] G. Grioli, M. Catalano, E. Silvestro, S. Tono, and A. Bicchi, "Adaptive synergies: An approach to the design of under-actuated robotic hands," IEEE Int. Conf. Intell. Robot. Syst., pp. 1251-1256, 2012.

[11] M. G. Catalano, G. Grioli, E. Farnioli, A. Serio, C. Piazza, and A. Bicchi, "Adaptive synergies for the design and control of the Pisa/IIT SoftHand," Int. J. Rob. Res., vol. 33, no. 5, pp. 768-782, Apr. 2014.

[12] C. Piazza, C. Della Santina, M. Catalano, G. Grioli, M. Garabini, and A. Bicchi, "SoftHand Pro-D: Matching dynamic content of natural user commands with hand embodiment for enhanced prosthesis control," in 2016 IEEE International Conference on Robotics and Automation (ICRA), 2016, pp. 3516-3523.

[13] M. Tavakoli and A. T. de Almeida, "Adaptive under-actuated anthropomorphic hand: ISR-SoftHand," in 2014 IEEE/RSJ International Conference on Intelligent Robots and Systems, 2014, pp. 1629-1634.

[14] M. Tavakoli, R. Batista, and L. Sgrigna, "The UC Softhand: Light Weight Adaptive Bionic Hand with a Compact Twisted String Actuation System," Actuators, vol. 5, no. 1, p. 1, Dec. 2015.
[15] M. Ciocarlie, F. M. Hicks, R. Holmberg, J. Hawke, M. Schlicht, J. Gee, S. Stanford, and R. Bahadur, "The Velo gripper: A versatile singleactuator design for enveloping, parallel and fingertip grasps," Int. J. Rob. Res., vol. 33, no. 5, pp. 753-767, Apr. 2014.

[16] S. B. Backus and A. M. Dollar, "An Adaptive Three-Fingered Prismatic Gripper With Passive Rotational Joints," IEEE Robot. Autom. Lett., vol. 1, no. 2, pp. 668-675, Jul. 2016.

[17] K. B. Shimoga and A. A. Goldenberg, "Soft robotic fingertips part I: A comparison of construction materials," Int. J. Rob. Res., vol. 15, no. 4, pp. 320-334, 1996.

[18] H. Takeuchi and T. Watanabe, "Development of a multi-fingered robot hand with softness-changeable skin mechanism," in Joint 41st International Symposium on Robotics and 6th German Conference on Robotics 2010, ISR/ROBOTIK 2010, 2010, vol. 1, pp. 606-612.

[19] K. C. Galloway, K. P. Becker, B. Phillips, J. Kirby, S. Licht, D. Tchernov, R. J. Wood, and D. F. Gruber, "Soft Robotic Grippers for Biological Sampling on Deep Reefs," Soft Robot., vol. 3, no. 1, pp. 2333, Mar. 2016.

[20] A. Pettersson, S. Davis, J. O. O. Gray, T. J. J. Dodd, and T. Ohlsson, "Design of a magnetorheological robot gripper for handling of delicate food products with varying shapes," J. Food Eng., vol. 98, no. 3, pp. $332-338,2010$

[21] R. Deimel and O. Brock, "A compliant hand based on a novel pneumatic actuator," in 2013 IEEE International Conference on Robotics and Automation, 2013, pp. 2047-2053.

[22] R. Deimel and O. Brock, "A novel type of compliant and underactuated robotic hand for dexterous grasping," Int. J. Rob. Res., vol. 35, no. 1-3, pp. 161-185, Jan. 2016

[23] F. Ilievski, A. D. Mazzeo, R. F. Shepherd, X. Chen, and G. M. Whitesides, "Soft Robotics for Chemists," Angew. Chemie Int. Ed., vol. 50, no. 8, pp. 1890-1895, Feb. 2011.

[24] A. Dameitry and H. Tsukagoshi, "Lightweight Underactuated Pneumatic Fingers Capable of Grasping Various Objects," in IEEE International Conference on Robotics and Automation, 2016, pp. 2009-2014.

[25] A. a Stokes, R. F. Shepherd, S. a Morin, F. Ilievski, and G. M. Whitesides, "A Hybrid Combining Hard and Soft Robots," Soft Robot., vol. 1, no. 1, pp. 70-74, Mar. 2014.

[26] J. Kim, A. Alspach, and K. Yamane, "3D printed soft skin for safe human-robot interaction," in 2015 IEEE/RSJ International Conference on Intelligent Robots and Systems (IROS), 2015, pp. 2419-2425.

[27] H. Choi, M. Koc, and M. Koç, "Design and feasibility tests of a flexible gripper based on inflatable rubber pockets," Int. J. Mach. Tools Manuf., vol. 46, no. 12-13, pp. 1350-1361, 2006.

[28] R. F. Shepherd, A. A. Stokes, R. M. D. Nunes, and G. M. Whitesides, "Soft Machines That are Resistant to Puncture and That Self Seal," Adv. Mater., vol. 25, no. 46, pp. 6709-6713, Dec. 2013.

[29] B. S. Homberg, R. K. Katzschmann, M. R. Dogar, and D. Rus, "Haptic identification of objects using a modular soft robotic gripper," in 2015 IEEE/RSJ International Conference on Intelligent Robots and Systems (IROS), 2015, pp. 1698-1705.

[30] E. Brown, N. Rodenberg, J. Amend, A. Mozeika, E. Steltz, M. R. Zakin, H. Lipson, and H. M. Jaeger, "Universal robotic gripper based on the jamming of granular material," Proc. Natl. Acad. Sci., vol. 107, no. 44, pp. 18809-18814, 2010.

[31] J. R. Amend, E. Brown, N. Rodenberg, H. M. Jaeger, and H. Lipson, "A Positive Pressure Universal Gripper Based on the Jamming of Granular Material," IEEE Trans. Robot., vol. 28, no. 2, pp. 341-350, Apr. 2012.

[32] C. Eppner and O. Brock, "Planning grasp strategies That Exploit Environmental Constraints," in 2015 IEEE International Conference on Robotics and Automation (ICRA), 2015, pp. 4947-4952.

[33] A. Holladay, R. Paolini, and M. T. Mason, "A general framework for open-loop pivoting," in 2015 IEEE International Conference on Robotics and Automation (ICRA), 2015, pp. 3675-3681.

[34] M. Toussaint, N. Ratliff, J. Bohg, L. Righetti, P. Englert, and S. Schaal, "Dual execution of optimized contact interaction trajectories," in 2014 IEEE/RSJ International Conference on Intelligent Robots and Systems, 2014, pp. 47-54.

[35] N. C. Dafle, A. Rodriguez, R. Paolini, B. Tang, S. S. Srinivasa, M. Erdmann, M. T. Mason, I. Lundberg, H. Staab, and T. Fuhlbrigge, "Extrinsic Dexterity : In-Hand Manipulation with External Forces," pp. $1578-1585,2014$

[36] D. E. Whitney, "Quasi-Static Assembly of Compliantly Supported Rigid Parts," J. Dyn. Syst. Meas. Control, vol. 104, no. 1, p. 65, 1982. 